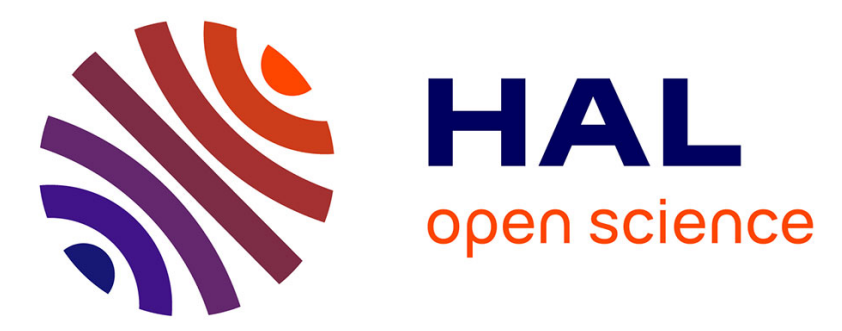

\title{
Macroscopic permeability of doubly porous materials with cylindrical and spherical macropores
}

\author{
Vincent Monchiet, Hai-Bang Ly, Daniel Grande
}

\section{To cite this version:}

Vincent Monchiet, Hai-Bang Ly, Daniel Grande. Macroscopic permeability of doubly porous materials with cylindrical and spherical macropores. Meccanica, 2019, 10.1007/s11012-019-01023-1 . hal02265596

\section{HAL Id: hal-02265596 \\ https://hal.science/hal-02265596}

Submitted on 18 Mar 2021

HAL is a multi-disciplinary open access archive for the deposit and dissemination of scientific research documents, whether they are published or not. The documents may come from teaching and research institutions in France or abroad, or from public or private research centers.
L'archive ouverte pluridisciplinaire $\mathbf{H A L}$, est destinée au dépôt et à la diffusion de documents scientifiques de niveau recherche, publiés ou non, émanant des établissements d'enseignement et de recherche français ou étrangers, des laboratoires publics ou privés. 


\title{
Macroscopic permeability of doubly porous materials with cylindrical and spherical macropores
}

\author{
${\text { Vincent } \text { Monchiet }^{\mathrm{a}} \text {, Hai-Bang Ly }}^{\mathrm{b}}$, Daniel Grande ${ }^{\mathrm{c}}$ \\ ${ }^{a}$ Université Paris-Est, Laboratoire Modélisation et Simulation Multi Echelle, \\ MSME UMR8208 CNRS, 5 boulevard Descartes, 77454 Marne la Vallée Cedex, \\ France \\ ${ }^{\mathrm{b}}$ University Of Transport Technology, Hanoi 100000, Vietnam \\ ${ }^{\mathrm{c}}$ Université Paris-Est, Institut de Chimie et des Matériaux Paris-Est, UMR 7182 \\ CNRS, 2 rue Henri Dunant, 94320 THiais, France
}

\begin{abstract}
We derivate analytic expressions for the effective permeability of a doubly porous medium which contains cylindrical or spherical macropores. The homogenization procedure is based on the consideration of a unit cell with a prescribed macroscopic pressure gradient or macroscopic velocity on its boundary. Within the unit cell, we have to solve the coupled Darcy-Stokes equations with appropriate interface models. The effective permeability of the bi-porous solid is determined by considering the composite cylinder and sphere assemblages. The solutions with prescribed macroscopic pressure gradient and velocity are provided and the influence in the choice of the interface model on the effective permeability of the doubly porous solid is studied. Finally, the results are compared with existing expressions provided in the literature and the estimate for the case of a cylindrical macropore is compared with finite element solutions.
\end{abstract}

Key words: Permeability, Homogenization, Cylindrical and Spherical Voids, Stokes, Darcy.

Email addresses: vincent.monchiet@univ-paris-est.fr (Vincent Monchiet), hai-bang.ly@univ-mlv.fr (Hai-Bang Ly), grande@icmpe.cnrs.fr (Daniel Grande). 


\section{Introduction}

Geomaterials such as concrete or rocks are naturally porous media and their transport properties are classically described by the Darcy law and the permeability coefficient. The derivation of the permeability in the framework of homogenization procedures has been largely documented in the literature using asymptotic approaches $[5,43,30]$ or volume average methods $[48,1]$. In the present paper, we focus on the permeability of a doubly porous material constituted of an initially porous structures containing non interconnected macropores. The apparition of the macropores is due to mechanical loading or chemical reactions and the determination of their role on the mass transfer properties is of particular interest for various applications such as fluid/gaz storage. Obviously, the geometry of fractures in geomaterials must be depicted by flat ellipsoids (see for instance Budiansky and O'Connell [17]), however, in this paper, we consider the case of cylindrical and spherical macropores by simplicity. The case of ellipsoidal shapes would be investigated in a next study. Note however that the results in the case of the spherical shape can be used to estimate the permeability of some bi-porous polymers (see for instance [31,32]).

The problem to study has three scales: the first is that of an interconnected network of submicron channels, the second scale is associated with the random distribution of non interconnected macropores, the last scale is the macroscopic one. The macroscopic permeability can be determined by a double homogenization approach along the lines of a method which has been derived for instance, by the asymptotic expansion method (see Auriault and Boutin [6-8], Royer et al. [40], Boutin et al. [11], Olny and Boutin [36]). At the intermediate scale, a coupled Darcy/Stokes problem has to be solved. However, the asymptotic method is not able to deliver the conditions et the interface between the Darcy region and the Stokes one. Two different approaches are generally considered in the literature: (i) the first uses the interface model of BeaversJoseph-Saffman (BJS) [10,41] (see also [35] for a historical background), (ii) the second one uses the Brinkman equation $[14,15]$. Some comparisons of these two approaches has been also provided on the literature for $2 \mathrm{~d}$ geometric configurations (see for instance [19]).

Analytic models has been first provided by Markov et al.[34] introducing the concept of an equivalent permeability for the macropores. The latter is determined by solving the coupled Darcy/Stokes equations with the BJS interface model for an isolated cavity embedded in an infinite porous matrix. The results are provided in the cases of a cylindrical or spherical pore. The results have been latter extended to the case of a spheroidal cavity by Rasoulzadeh et al. [39] which can mimic fractures by taking an oblate spheroid with a small value of the aspect ratio. Another analytic solutions has been derived by Silva and Ginzburg [44] for a composite cylinder, i.e. two concentric cylinders in which the flow obeys to the Brinkman equation but with different coefficients in the core and in the coating. 
In the present paper, we derive the effective permeability of bi-porous materials with cylindrical and spherical macropores by using the approach based on Darcy-Stokes equations. The influence in the choice of the interface model on the effective permeability is then analyzed. The effective permeability is determined by considering the composite cylinder and sphere models which have been often considered by the past by various authors in the context of porous media $[23,27,45,12]$. The analytic solutions with prescribed macroscopic pressure gradient or macroscopic velocity at the boundary of the representative volume element are provided and the close form expression of the effective permeability is given. The solutions are compared with finite element ones in the case of a cylindrical shape. We also determine the equivalent permeability of the Stokes region and it is compared with the results of Markov et al. [34].

\section{Homogenization problem}

\subsection{Local equations and interface conditions}

Consider a Representative Volume Element (RVE) of a doubly porous solid. Both the porous solid and the macropores are fulfilled by a Newtonian viscous fluid with the dynamic viscosity $\mu$. We denote by $\Omega_{s}$ the domain occupied by the porous solid and by $\Omega_{f}$ the domain of the pores. Obviously, the permeability of the solid phase is associated with an interconnected porous microstructure at a lower scale (see figure 1 ). The permeability of the matrix is assumed to be known (obtained from experiments) and the macropores (the first porosity) are assumed to be non interconnected. Denoting by $h$ the characteristic length of the channels of the initial interconnected microporous solid (the second porosity), we assume that $h$ is sufficiently small compared to cavities radius $a$ such that there is a scale separation between the two porosities. The macropores are then embedded in a homogenous porous material having the permeability $k$.

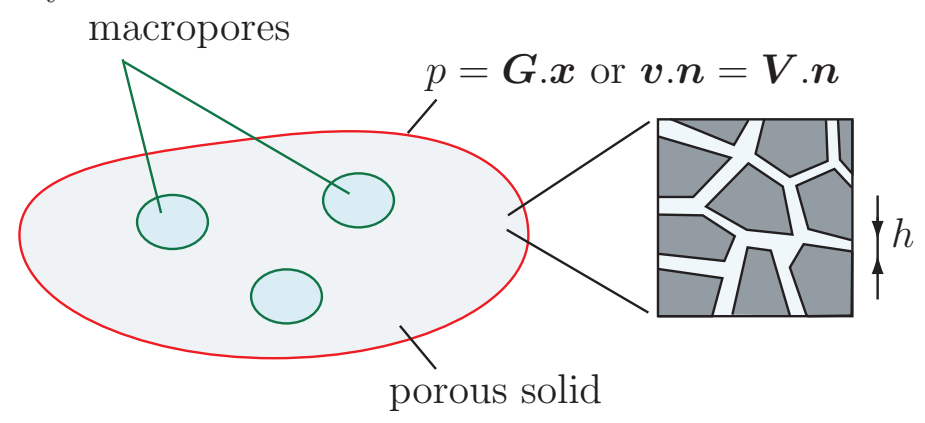

Fig. 1. Schematic representation of the RVE

In the macropores, the fluid flow is described by the Stokes equations: 


$$
\begin{array}{rlrl}
\mu \Delta \boldsymbol{v}-\nabla p=0 & \forall \boldsymbol{x} \in \Omega_{f} \\
\operatorname{div} \boldsymbol{v}=0 & \boldsymbol{x} \in \forall \Omega_{f}
\end{array}
$$

Within the solid, the fluid flow obeys to the Darcy law with the incompressibility condition:

$$
\begin{array}{cc}
\boldsymbol{v}=-\frac{k}{\mu} \nabla p & \forall \boldsymbol{x} \in \Omega_{s} \\
\operatorname{div} \boldsymbol{v}=0 & \forall \boldsymbol{x} \in \Omega_{s}
\end{array}
$$

At the interface between the fluid and the solid, the Beaver-Joseph (BJ) [10] model is often used:

$$
\begin{aligned}
\boldsymbol{v}^{(s)} \cdot \boldsymbol{\nu}=\boldsymbol{v}^{(f)} \cdot \boldsymbol{\nu} & \forall \boldsymbol{x} \in \Gamma \\
2 \mu \boldsymbol{\nu} \cdot \boldsymbol{d}\left(\boldsymbol{v}^{(f)}\right) \cdot \boldsymbol{\tau}=-\alpha\left[\boldsymbol{v}^{(f)}-\boldsymbol{v}^{(s)}\right] . \boldsymbol{\tau} & \forall \boldsymbol{x} \in \Gamma \\
2 \mu \boldsymbol{\nu} \cdot \boldsymbol{d}\left(\boldsymbol{v}^{(f)}\right) . \boldsymbol{\nu}=p^{(f)}-p^{(s)} & \forall \boldsymbol{x} \in \Gamma
\end{aligned}
$$

in which $p^{(f)}, \boldsymbol{v}^{(f)}$ and $p^{(s)}, \boldsymbol{v}^{(s)}$ represents the pressure and velocity fields taken at each side of the interface $\Gamma$, exponent $(s)$ makes reference to the porous solid and $(f)$ to the fluid phase (i.e. the macropores). Also, $\boldsymbol{\nu}$ and $\boldsymbol{\tau}$ represent the normal and the tangential unit vectors acting on $\Gamma$. In equation (6), $\alpha$ is a coefficient of the BJ model, $\boldsymbol{d}\left(\boldsymbol{v}^{(f)}\right)$ represents the strain rate tensor computed in the fluid region. These interface conditions represent: the continuity of the mass flux (5), the discontinuity for the tangential velocity (6) and the continuity for the normal traction (7). At the interface $\Gamma$, the normal component of the velocity field is then continuous but the tangential component and the pressure are discontinuous.

Based on their experimental observations, Beaver and Joseph [10] proposed the following expressions for the coefficient $\alpha$ :

$$
\alpha=\frac{\mu}{\sqrt{k}} \delta
$$

in which $\delta$ is a dimensionless coefficient called slip coefficient and is characteristic of the geometry of the porous solid. This coefficient has been determined experimentally by Beavers and Joseph [10] for Nickel foametal and found 0.78, 1.45 and 4.0 for porous microstructures having average pore size of $0.016,0.034$ and 0.045 inches respectively. For Aloxite they found a value of 0.1 with the average pore size of 0.013 and 0.027 inches. Numerical studies have been also provided by Sahraoui and Kaviany [42] for $2 \mathrm{~d}$ periodic structure made of cylindrical particles and found a value of the slip coefficient closed to 2 .

Some other theoretical support of the BJ model has been provided in the literature. For instance, Saffman [41] uses a statical treatment and found that 
the tangential velocity component in the porous solid could be neglected and suggests to use the following condition for the tangential velocity:

$$
2 \mu \boldsymbol{\nu} \cdot \boldsymbol{d}\left(\boldsymbol{v}^{(f)}\right) \cdot \boldsymbol{\tau}=-\alpha \boldsymbol{v}^{(f)} \cdot \boldsymbol{\tau} \quad \boldsymbol{x} \in \Gamma
$$

This form has been considered in numerous works (see for instance $[28,4,46]$ ). A tentative to demonstrate the BJS interface model has been provided by Jager and Mikelic [24] using the asymptotic series expansion method and a scale separation. Moreover, the authors show that the slip coefficient could be determined through an auxiliary-layer type problem.

Auriault [9] analyses the interface condition at the frontier between a fluid and a porous solid by the method of asymptotic series expansion and conclude that the adherence condition could be used at the frontier between the Darcy and the Stokes region:

$$
\boldsymbol{v}^{(f)} \cdot \boldsymbol{\tau}=0 \quad \boldsymbol{x} \in \Gamma
$$

The adherence condition is a particular case of the BJS model corresponding to $\alpha=+\infty$.

In order to avoid in confusion, the equations (5), (6) and (7) are called Beavers and Joseph (BJ) conditions. We call Beavers-Joseph-Saffman (BJS) the interface model which use conditions (5), (9) and (7). The adherence model corresponds to conditions (5), (10) and (7).

For all the calculations provided in the next sections, it is preferable to use the following unique equation for the jump of the tangential velocity:

$$
2 \mu \boldsymbol{\nu} \cdot \boldsymbol{d}\left(\boldsymbol{v}^{(f)}\right) \cdot \boldsymbol{\tau}=-\alpha\left[\boldsymbol{v}^{(f)}-\epsilon \boldsymbol{v}^{(s)}\right] . \boldsymbol{\tau} \quad \boldsymbol{x} \in \Gamma
$$

where $\epsilon=1$ and $\alpha$ is given by (8) for the BJ model, $\epsilon=0$ and $\alpha$ is given by (8) for the BJS model while $\alpha=+\infty$ and $\epsilon=0$ for the adherence condition. Note that other interfacial conditions has been proposed in the literature (see for instance $[37,38,47])$ but are not considered in the present study. Note that the existence and uniqueness of the solution of Darcy/Stokes coupled problem with the BJS interface condition has been studied for instance by Arbogast and Lehr [3]. Note also that the velocity field is divergence free in the porous solid and in the micro-pores. The pressure is then an harmonic function everywhere in the RVE:

$$
\Delta p=0 \quad \forall \boldsymbol{x} \in \Omega_{s} \cup \Omega_{f}
$$




\subsection{Boundary conditions and macroscopic permeability}

In order to determine the macroscopic permeability of the porous material, let $\Omega$ be subjected to the following prescribed pressure on its external surface $\partial \Omega$ :

$$
p=\boldsymbol{G} \cdot \boldsymbol{x}, \quad \forall \boldsymbol{x} \in \partial \Omega
$$

or the prescribed normal velocity:

$$
\boldsymbol{v} . \boldsymbol{n}=\boldsymbol{V} . \boldsymbol{n}, \quad \forall \boldsymbol{x} \in \partial \Omega
$$

where $\boldsymbol{G}$ represents the macroscopic pressure gradient, $\boldsymbol{V}$ represents the macroscopic velocity and $\boldsymbol{n}$ the normal unit vector taken on the boundary $\partial \Omega$.

A velocity field is said admissible if it is divergence free in $\Omega_{f}$ and in $\Omega_{s}$, if the normal velocity $\boldsymbol{v} . \boldsymbol{\nu}$ is continuous across $\Gamma$ and if $\boldsymbol{v}$ satisfies to the boundary condition (14). For such velocity fields, we have the compatibility condition:

$$
\boldsymbol{V}=<\boldsymbol{v}>_{\Omega}
$$

that is easily proved by using the divergence theorem.

A pressure field is said admissible if it is continuous and continuously differentiable in each phase and satisfy to the boundary condition (13). For such pressure field, we have the following compatibility condition:

$$
\boldsymbol{G}=<\nabla p>_{\Omega}+\frac{1}{|\Omega|} \int_{\Gamma}[p] \boldsymbol{\nu} d x
$$

where $[p]=p^{(s)}-p^{(f)}$ represents the jump of $p$ across $\Gamma$. The above condition extends the classic average relation in the case of non continuous fields. For instance, equivalent relation also appears for the average of stress in the case of elastic composites with imperfect interfaces (see for instance [29]).

If the boundary condition (13) is applied to the RVE, due to the linearity of the Darcy-Stokes equations, the local velocity and pressure fields linearly depends on the applied macroscopic pressure gradient $\boldsymbol{G}$. This mean that there exist a localization tensor $\boldsymbol{A}(\boldsymbol{x})$ such that:

$$
\boldsymbol{v}=\boldsymbol{A}(\boldsymbol{x}) \cdot \boldsymbol{G}
$$

Introducing this expression in the average relation (15), it leads to:

$$
\boldsymbol{V}=-\frac{1}{\mu} \boldsymbol{K}^{h o m} \cdot \boldsymbol{G}
$$

where we have introduced the macroscopic permeability:

$$
\boldsymbol{K}^{h o m}=-\mu<\boldsymbol{A}(\boldsymbol{x})>_{\Omega}
$$


Alternatively, if the boundary condition (14) is used, the local pressure gradient can be expressed as function of $\boldsymbol{V}$ by introducing the localization tensor $\boldsymbol{B}(\boldsymbol{x})$ :

$$
p=\boldsymbol{B}(\boldsymbol{x}) \cdot \boldsymbol{V}
$$

Introducing this expression in (16), it leads to:

$$
\left[\boldsymbol{K}^{h o m}\right]^{-1}=-\frac{1}{\mu}\left\{<\nabla \boldsymbol{B}(\boldsymbol{x})>_{\Omega}+\frac{1}{|\Omega|} \int_{\Gamma} \boldsymbol{\nu} \stackrel{s}{\otimes}[\boldsymbol{B}(\boldsymbol{x})] d x\right\}
$$

The value of $\boldsymbol{K}^{\text {hom }}$ is different from the local permeability $k$ due to the presence of the macropores. Obviously, when the volume of the macropores is null, the RVE is only constituted of the porous medium with the isotropic permeability $k$ and the macroscopic permeability is $\boldsymbol{K}^{\text {hom }}=k \boldsymbol{I}$. The main objective of this work is to determine the homogenized permeability tensor $\boldsymbol{K}^{\text {hom }}$ as function of the local permeability $k$, the macro-pores radius $a$ and of the volume fraction $f$. Also the choice of the interface model on the effective permeability is investigated considering the BJ, BJS or the adherence interface model.

\section{Exact solution for a cylindrical macropore}

In this section, we aim to derive exact solutions of the Darcy-Stokes problem in the case of a unit cell containing a single cylindrical pore. The cylinder is made up of an isotropic porous solid with the permeability $k$ containing a macropore of radius a fulfilled by a viscous fluid which obeys to the Stokes equations. At its external boundary, the pressure $p=\boldsymbol{G} \cdot \boldsymbol{x}$ is applied. The external radius is denoted by $b$.

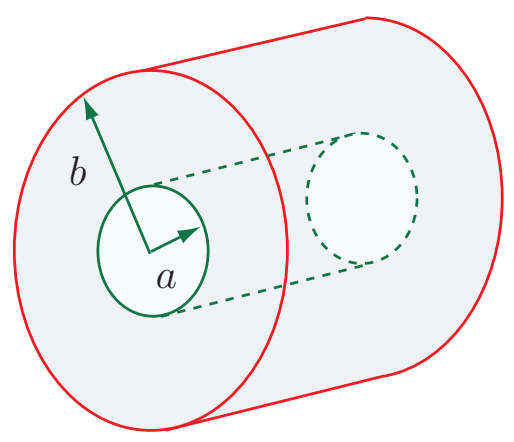

Fig. 2. Hollow cylinder with external radius $b$ containing a cylindrical void of radius $a$.

Regarding the boundary condition on the porous cylinder, it reads in the cylindrical frame $(r, \theta, z)$ :

$$
p=G_{1} b \cos (\theta)+G_{2} b \sin (\theta)
$$


The solution with a prescribed normal velocity at the boundary of the RVE is provided in the appendix A.1. It leads to the same expression for the macroscopic permeability.

We assume that only the component $G_{1}$ is non null. In order to satisfy the boundary condition (22), we choose the following expression for the local pressure:

$$
p=F(r) \cos (\theta)
$$

in the fluid and in the solid.

Obviously, the function $F(r)$ takes different expressions in the solid and in the fluid regions. The pressure being a harmonic function, it leads to:

$$
\Delta p=\frac{\partial^{2} p}{\partial r^{2}}+\frac{1}{r} \frac{\partial p}{\partial r}+\frac{1}{r^{2}} \frac{\partial^{2} p}{\partial \theta^{2}}=\left[F^{\prime \prime}(r)+\frac{1}{r} F^{\prime}(r)-\frac{1}{r^{2}} F(r)\right] \cos (\theta)=0
$$

which is satisfied for:

$$
F(r)=\left\{\begin{array}{l}
A_{1} r+\frac{B_{1}}{r} \text { in the solid } \\
A_{2} r+\frac{B_{2}}{r} \text { in the fluid }
\end{array}\right.
$$

where $A_{1}, B_{1}, A_{2}$ and $B_{2}$ are four constants. The term $B_{2} / r$ is eliminated since it diverges at $r=0$. The velocity field in the solid is given by the Darcy law:

$$
\begin{array}{r}
v_{r}=-\frac{k}{\mu} F^{\prime}(r) \cos (\theta)=-\frac{k}{\mu}\left[A_{1}-\frac{B_{1}}{r^{2}}\right] \cos (\theta) \\
v_{\theta}=\frac{k}{\mu} \frac{F(r)}{r} \sin (\theta)=\frac{k}{\mu}\left[A_{1}+\frac{B_{1}}{r^{2}}\right] \sin (\theta)
\end{array}
$$

In the macropore, the velocity field must comply with the Stokes equations, which reads, in the cylindrical frame:

$$
\begin{aligned}
& \mu \Delta v_{r}-\frac{\mu}{r^{2}}\left[2 \frac{\partial v_{\theta}}{\partial \theta}+v_{r}\right]-F^{\prime}(r) \cos (\theta)=0 \\
& \mu \Delta v_{\theta}+\frac{\mu}{r^{2}}\left[2 \frac{\partial v_{r}}{\partial \theta}-v_{\theta}\right]+\frac{F(r)}{r} \sin (\theta)=0
\end{aligned}
$$

This suggests to use the following expressions for the components of the velocity field:

$$
v_{r}=G(r) \cos (\theta), \quad v_{\theta}=H(r) \sin (\theta)
$$

The introduction of these expressions in equations (28) and (29) leads to a linear system of differential equations for $G(r)$ and $H(r)$ which have the following general solution: 


$$
G(r)=\frac{A_{2}}{8 \mu} r^{2}+C_{2}+\frac{D_{2}}{r^{2}}, \quad H(r)=-\frac{3 A_{2}}{8 \mu} r^{2}-C_{2}+\frac{D_{2}}{r^{2}}
$$

where $C_{2}$ and $D_{2}$ are two additional constants. Again, the term $D_{2} / r^{2}$ is eliminated since it diverges at $r=0$.

The interface conditions must now be verified. The continuity of the flux reads:

$$
v_{r}^{(f)}(r=a)=v_{r}^{(s)}(r=a)
$$

The interface condition for the tangential velocity is:

$$
\mu\left[\frac{1}{r} \frac{\partial v_{r}^{(f)}}{\partial \theta}-\frac{v_{\theta}^{(f)}}{r}+\frac{\partial v_{\theta}^{(f)}}{\partial r}\right]_{(r=a)}=-\alpha\left[v_{\theta}^{(f)}-\epsilon v_{\theta}^{(s)}\right]_{(r=a)}
$$

The continuity of the normal traction is:

$$
2 \mu\left[\frac{\partial v_{r}^{(f)}}{\partial r}\right]_{(r=a)}=\left[p^{(f)}-p^{(s)}\right]_{(r=a)}
$$

and finally the boundary condition for the pressure is satisfied if:

$$
A_{1} b+\frac{B_{1}}{b}=G_{1} b
$$

Introducing expressions (26), (27) and (30) with (31) in Eqs. (32) to (34) leads to a linear set of algebraic equations for the unknown coefficients. The solution of this linear system is:

$$
\left.\begin{array}{l}
A_{1}=\frac{G_{1}}{Q}\left[2 a \mu+\alpha a^{2}+2 \alpha(1+\epsilon) k\right] \\
B_{1}=-\frac{G_{1}}{Q}\left(2 \mu a+\alpha a^{2}-2 \alpha k(1-\epsilon)\right) a^{2} \\
A_{2}=\frac{8 k \alpha G_{1}}{Q} \\
C_{2}=-\frac{k G_{1}}{\mu Q}\left(4 \alpha \epsilon k+4 a \mu+3 \alpha a^{2}\right)
\end{array}\right\}
$$

in which $Q$ is given by:

$$
Q=\left[\alpha a^{2}+2 a \mu+2 \alpha \epsilon k\right](1-f)+2 \alpha k(1+f)
$$




\section{Exact solutions for spherical macropore}

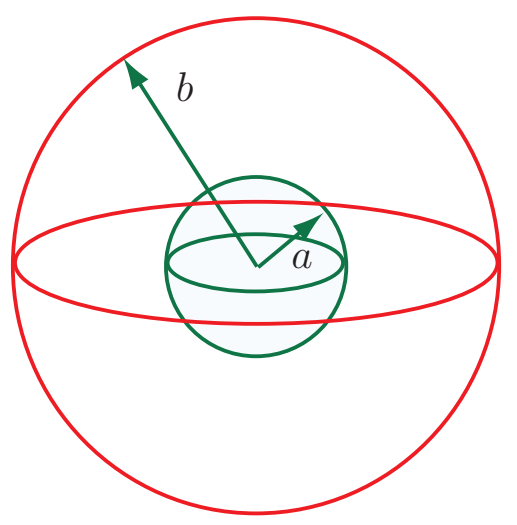

Fig. 3. Hollow sphere with external radius $b$ containing a spherical void of radius $a$.

In this section, we consider the case of a spherical cell of radius $b$ containing a macropore of radius $a$. The permeability of the porous matrix is still denoted by $k$ and the same methodology follows in the case of a cylindrical macropore is still considered. The boundary condition (13) reads in the spherical frame $(r, \theta, \varphi)$ :

$$
\left.p=G_{1} r \cos (\theta) \sin (\varphi)+G_{2} r \sin \theta\right) \sin (\varphi)+G_{3} r \cos (\varphi)
$$

The solution with a prescribed normal velocity at the boundary of the RVE is provided in the appendix A.2. Again, the same expression for the macroscopic permeability is obtained with this kind of boundary conditions.

Due to the symmetry of the problem, only the case $G_{1} \neq 0$ and $G_{2}=G_{3}=0$ is considered. The verification of the boundary condition suggests to use the following expression for the pressure:

$$
p=F(r) \cos (\theta) \sin (\varphi)
$$

The pressure being harmonic, we deduce that the function $F(r)$ has the following expression:

$$
F(r)= \begin{cases}A_{1} r+\frac{B_{1}}{r^{2}} & \text { in the solid } \\ A_{2} r & \text { in the fluid }\end{cases}
$$

In the solid matrix, the velocity is given by the Darcy law: 


$$
\begin{array}{r}
v_{r}=-\frac{k}{\mu}\left[A_{1}-\frac{2 B_{1}}{r^{3}}\right] \cos (\theta) \sin (\varphi) \\
v_{\theta}=-\frac{k}{\mu}\left[A_{1}+\frac{B_{1}}{r^{3}}\right] \sin (\theta) \\
v_{\varphi}=-\frac{k}{\mu}\left[A_{1}+\frac{B_{1}}{r^{3}}\right] \cos (\theta) \cos (\varphi)
\end{array}
$$

In the fluid phase, we use the following expressions for the velocity field:

$$
\begin{array}{r}
v_{r}=G_{1}(r) \cos (\theta) \sin (\varphi) \\
v_{\theta}=G_{2}(r) \sin (\theta) \\
v_{\varphi}=G_{3}(r) \cos (\theta) \cos (\varphi)
\end{array}
$$

The verification of the Stokes equations leads to:

$$
\begin{array}{r}
G_{1}(r)=C_{1}+C_{2} r^{2}+\frac{C_{3}}{r}+\frac{C_{4}}{r^{3}} \\
G_{2}(r)=G_{3}(r)=\frac{A_{2}}{4 \mu} r^{2}+C_{1}-\frac{C_{2}}{2} r^{2}+\frac{C_{3}}{r}-\frac{C_{4}}{2 r^{3}}
\end{array}
$$

This field is divergence free for $C_{2}=A_{2} /(10 \mu)$.

Obviously, the term in " $1 / r "$ and " $1 / r^{3} "$ are discarded since they diverge à $r=0$. Finally, the constants are determined with the boundary condition at $r=b$ and the interface conditions:

$$
\begin{array}{r}
\mu\left[\frac{1}{r \sin (\varphi)} \frac{\partial v_{r}^{(f)}}{\partial \theta}-\frac{v_{\theta}^{(f)}}{r}+\frac{\partial v_{\theta}^{(f)}}{\partial r}\right]_{(r=a)}=-\alpha\left[v_{\theta}^{(f)}-\epsilon v_{\theta}^{(s)}\right]_{(r=a)}^{(f)}(r=a)=v_{r}^{(s)}(r=a) \\
\mu\left[\frac{1}{r} \frac{\partial v_{r}^{(f)}}{\partial \varphi}-\frac{v_{\varphi}^{(f)}}{r}+\frac{\partial v_{\varphi}^{(f)}}{\partial r}\right]_{(r=a)}=-\alpha\left[v_{\varphi}^{(f)}-\epsilon v_{\varphi}^{(s)}\right]_{(r=a)}, \\
2 \mu\left[\frac{\partial v_{r}^{(f)}}{\partial r}\right]_{(r=a)}=\left[p^{(f)}-p^{(s)}\right]_{(r=a)}
\end{array}
$$

Equations (50) and (51) correspond to the discontinuity of the tangential velocity components, along direction $\boldsymbol{e}_{\theta}$ and $\boldsymbol{e}_{\varphi}$ respectively. These conditions have found to be equivalent since $G_{2}(r)=G_{3}(r)$.

Additionally, the boundary condition for the pressure is satisfied for:

$$
A_{1} b+\frac{B_{1}}{b^{2}}=G_{1} b
$$

The identification of the constants leads to: 


$$
\begin{array}{r}
A_{1}=\frac{G_{1}\left(a^{2} \alpha+6 \alpha k(2+\epsilon)+3 a \mu\right)}{Q}, \\
B_{1}=-\frac{G_{1} a^{3}}{Q}\left(a^{2} \alpha+3 a \mu-6 \alpha(1-\epsilon) k\right), \\
C_{1}=-\frac{3 G_{1} k}{\mu Q}\left(3 a \mu+2 a^{2} \alpha+6 \alpha \epsilon k\right), \\
A_{2}=\frac{30 G_{1} \alpha k}{Q}
\end{array}
$$

with:

$$
Q=\left[\alpha a^{2}+3 a \mu+6 k \alpha \epsilon\right](1-f)+6 \alpha k(2+f)
$$

\section{$5 \quad$ Effective permeability}

\subsection{Analytic solution for cylindrical/spherical macropores}

The macroscopic velocity is computed from the average rule (15). The in-plane permeability is $\boldsymbol{K}^{\text {hom }}=K^{\text {hom }} \boldsymbol{I}_{2}$ where $\boldsymbol{I}_{2}$ is the identity in $2 \mathrm{~d}$ dimension. Expressions of $K^{\text {hom }}$ are:

$$
\begin{array}{ll}
K^{\text {hom }}=k\left\{1+2 f \frac{\delta+2 \sqrt{\kappa}}{[\delta+2 \sqrt{\kappa}](1-f)+4 \delta \kappa}\right\} & \text { BJ model } \\
K^{\text {hom }}=k\left\{1+2 f \frac{\delta(1-2 \kappa)+2 \sqrt{\kappa}}{[\delta+2 \sqrt{\kappa}](1-f)+2 \delta \kappa(1+f)}\right\} & \text { BJS model } \\
K^{\text {hom }}=k\left\{1+2 f \frac{1-2 \kappa}{1-f+2 \kappa(1+f)}\right\} & \text { Adherence model }
\end{array}
$$

The dimensionless coefficient $\kappa$ is defined by:

$$
\kappa=\frac{k}{a^{2}}
$$

and the dimensionless coefficient $\delta$ has been introduced in equation (8). Coefficient $\kappa$ accounts for the macropores size.

In the case of a spherical macropore, the permeability tensor is isotropic, $\boldsymbol{K}^{\text {hom }}=K^{\text {hom }} \boldsymbol{I}$ where $K^{\text {hom }}$ is defined by: 


$$
\begin{array}{ll}
K^{\text {hom }}=k\left\{1+3 f \frac{\delta+3 \sqrt{\kappa}}{[\delta+3 \sqrt{\kappa}](1-f)+18 \kappa \delta)}\right\} & \text { BJ model } \\
K^{h o m}=k\left\{1+3 f \frac{\delta(1-6 \kappa)+3 \sqrt{\kappa}}{[\delta+3 \sqrt{\kappa}](1-f)+6 \kappa \delta(2+f))}\right\} & \text { BJS model } \\
K^{\text {hom }}=k\left\{1+3 f \frac{1-6 \kappa}{1-f+6 \kappa(2+f)}\right\} & \text { Adherence model }
\end{array}
$$

Considering coefficient $\kappa$, it depends on the permeability $k$ of the microporous matrix and on the radius of the macropores. For geomaterials, the permeability is typically inferior to $10^{-12} \mathrm{~m}^{2}$ (i.e. one Darcy). Assuming that the macropores radius is superior to $10^{-6} \mathrm{~m}$, we deduce that the value of $\kappa$ is strictly inferior to 1 . The value of $\kappa$ can reach very small values, considering for instance a geomaterial with very low permeability $\left(k=10^{-18} \mathrm{~m}^{2}\right.$ for example) and macropores of $10 \mu \mathrm{m}$, it gives $\kappa=10^{-8}$.

From a homogenization point of view, it is raisonnable to suppose that $\kappa$ is a small parameter. Indeed, the permeability of the porous matrix is $k=O\left(h^{2}\right)$ where $h$ is the characteristic length of the first (sub-micron) porosity, then $\kappa=O\left(h^{2} / a^{2}\right)$. Moreover, $h<<a$ since we assumed a separation of scales between the two porosities. Assuming that $h / a<0.1$, that is the limit for having the scale separation, we deduce that $\kappa \leq 10^{-2}$. Consequently, in all the applications proposed in the paper, we limit the value of $\kappa$ by $10^{-2}$.

Regarding expression (59), the macroscopic permeability decreases with $f$ if:

$$
\delta(1-2 \kappa)+2 \sqrt{\kappa}<0
$$

and regarding expression (59), if:

$$
\kappa>\frac{1}{2}
$$

This obviously leading to non physical results since it is expected that the presence of the macropores increases the effective permeability. Similar conditions could be derived for the spherical macropore. As mentioned above, it is reasonable to assume that $\kappa$ is small and then the above conditions are never attained. It must be mentioned that the effective permeabilities derived with the BJ interface model are monotonously increasing functions with the porosity $f$ whatever the value of $\delta$ and $\kappa$.

On figure 4, we represent the variations of the dimensionless permeability $K^{h o m} / k$ for a cylindrical macropore as function of the porosity $f$ and for different values of the slip coefficient $\delta$ and parameter $\kappa$. On figure 5 , we display the same results but for the spherical macropore.

It is observed, for the cylindrical macropore, that the effective permeability obtained with the three interface models leads to the same estimation. There is slight differences when considering the adherence model instead of the BJ or BJS models when $\delta=0.1$ and $\kappa=10^{-2}$. In the case of the spherical macropore, the three interface models leads to the same estimate when $\kappa=10^{-4}$. 
However, when $\kappa=10^{-2}$, the estimate obtained with the adherence model differs from two others. As a first conclusion, we observe that the BJ and BJS interface models leads to the same estimates for the effective permeability for any values of $\kappa$ and $\delta$. Consequently, the hypothesis formulated by Saffman [41], which supposes that the tangential components of the velocity field in the Darcy region is negligible compared to its counterpart in the Stokes region, is verified for microstructures with isolated spherical and cylindrical macropores.
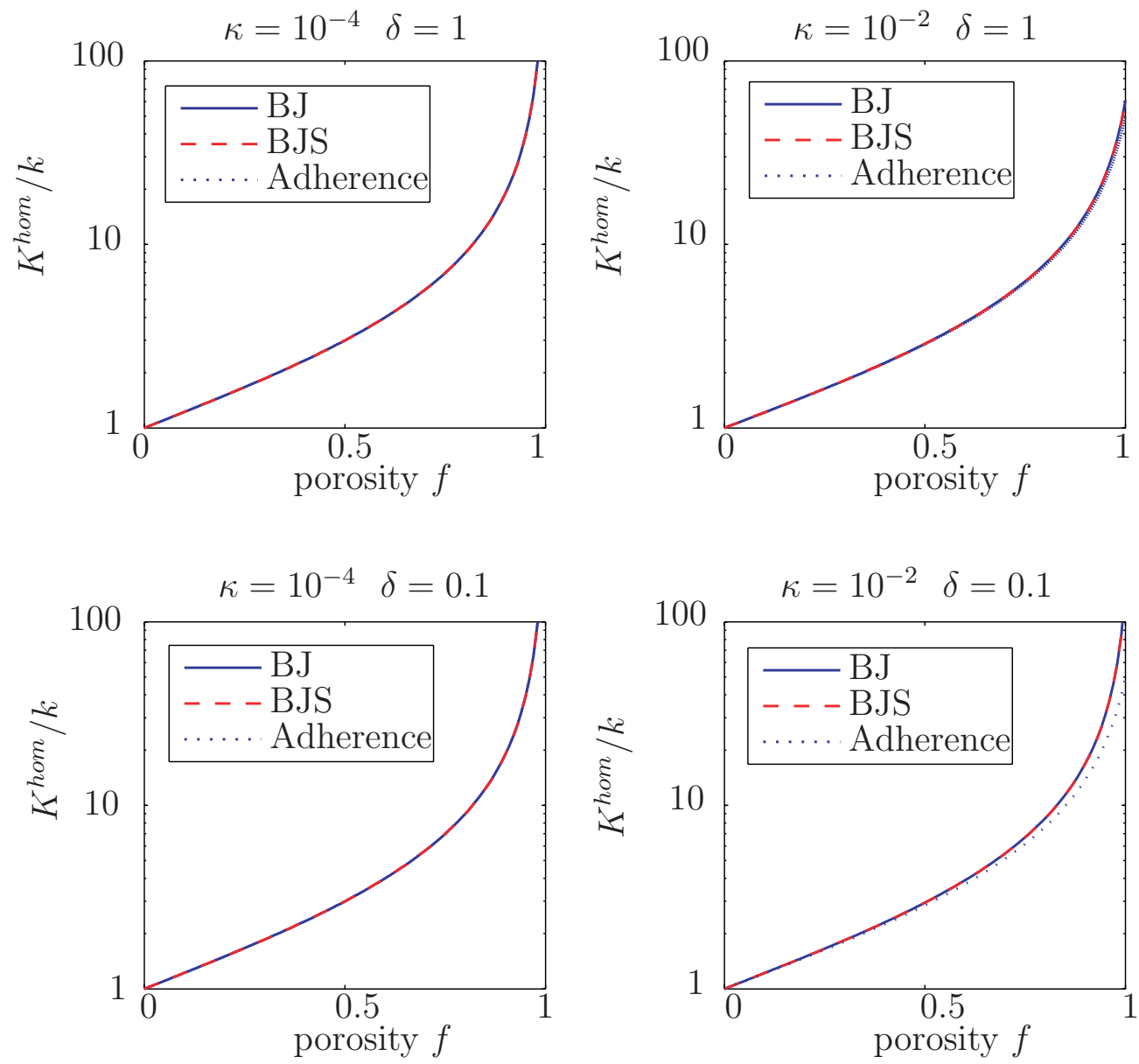

Fig. 4. Case of a cylindrical macropore. Variations of the dimensionless permeability $K^{\text {hom }}$ with the porosity $f$ for $\delta=1$ and $\kappa=1 e-4$. 

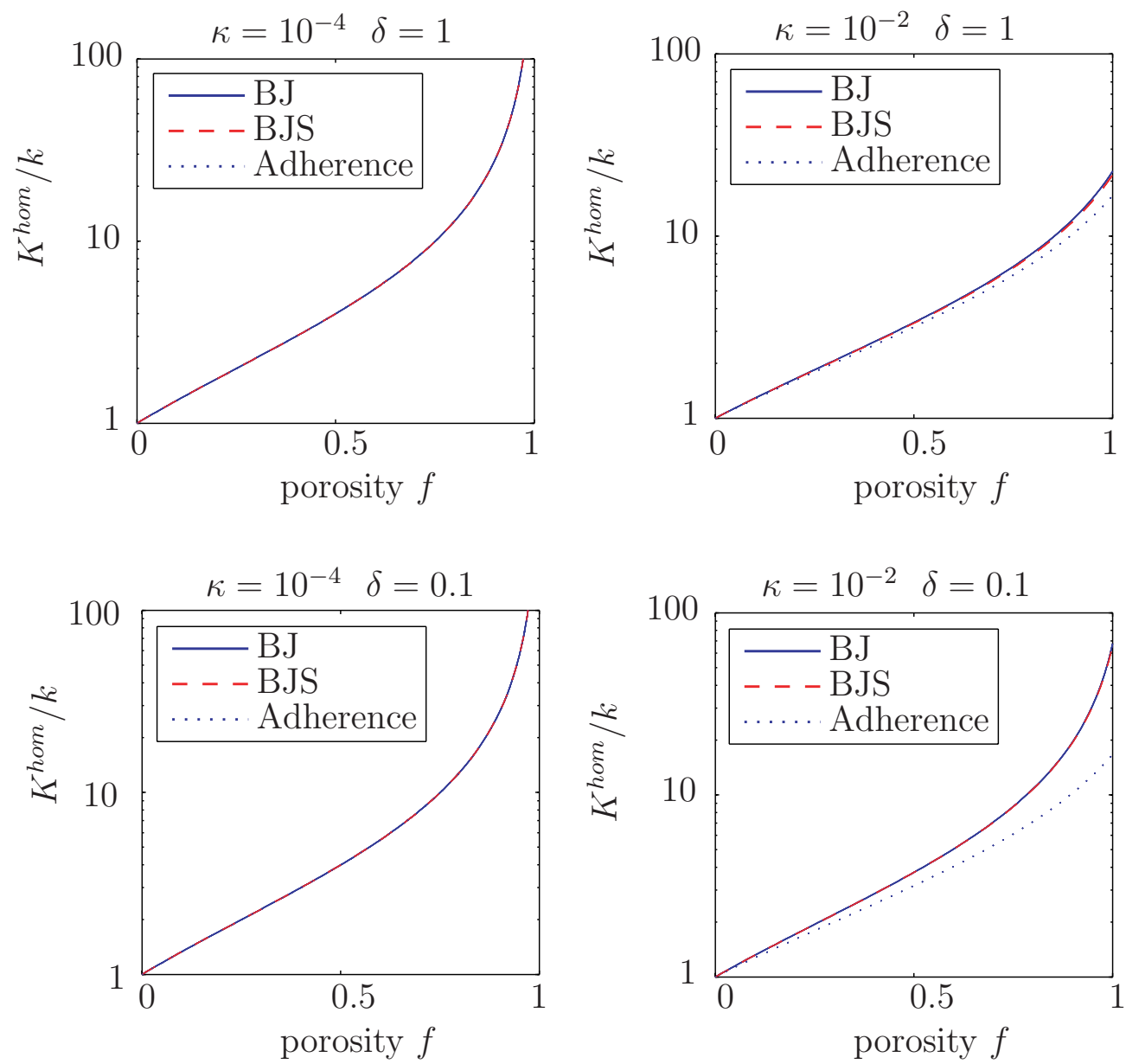

Fig. 5. Case of a spherical macropore. Variations of the dimensionless permeability $K^{h o m}$ with the porosity $f$ for $\delta=1$ and $\kappa=1 e-2$.

The variations of the effective permeability with the coefficient $\kappa$ is now provided in Fig. 6, 7 and 8 for the slip coefficient values $\delta=0.01, \delta=1$ and $\delta=100$ respectively. The porosity if $f=0.5$. On each figure, it can be observed that the three models (BJ, BJS and adherence) are equivalent at small values of $\kappa$ (typically inferior to $10^{-4}$ ). Moreover the estimate reach a plateau for very small values of $\kappa$. When the slip coefficient is small (see for instance the case $\delta=0.01$ on Fig. 6), the estimates obtained with the BJ and BJS interface conditions are equivalent but are very different to that derived with the adherence condition. The latter provides a lower estimate for the effective permeability in the range $10^{-3} \leq \kappa \leq 1$. Moreover the dependence of $K^{\text {hom }} / k$ with $\kappa$ is important for the adherence but less significant with the BJ and BJS interface conditions. At an intermediate value of $\delta$ (see for instance the case $\delta=1$ on Fig. 7). The effect of $\kappa$ on the effective permeability is more prominent for the BJ and BJS models than in the case $\delta=0.01$. Also, the three estimates are different in the range $10^{-3} \leq \kappa \leq 1$. This suggests that the tangential velocity in the microporous solid (that is neglected in the BJS model) 
is no longer negligible compared to the tangential velocity in the fluid region. For large values of the slip coefficient (see for instance the case $\delta=100$ on Fig. 8), the permeability with the BJS and adherence conditions are equivalent but differs from that which uses the BJ interface condition.

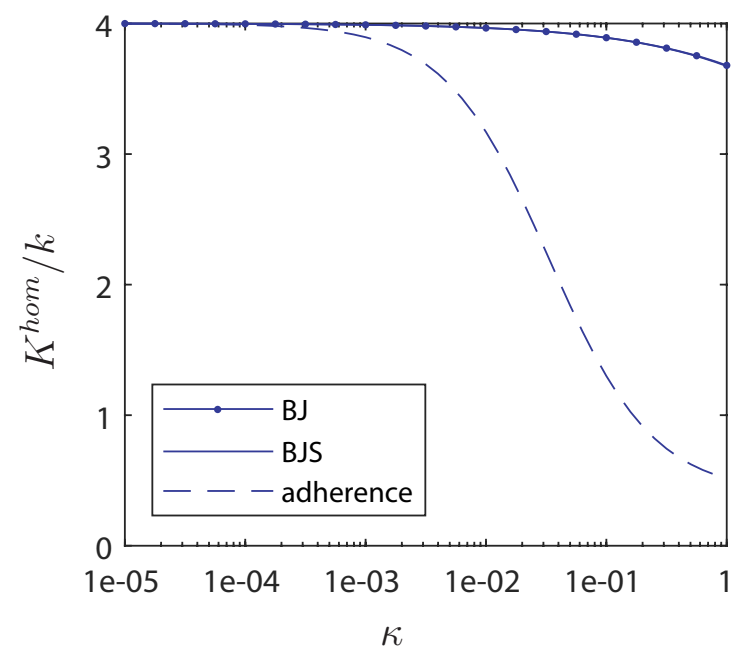

Fig. 6. Case of a spherical macropore. Variations of the dimensionless permeability $K^{\text {hom }} / k$ with $\kappa$ for $\delta=0.01$ and $f=0.5$.

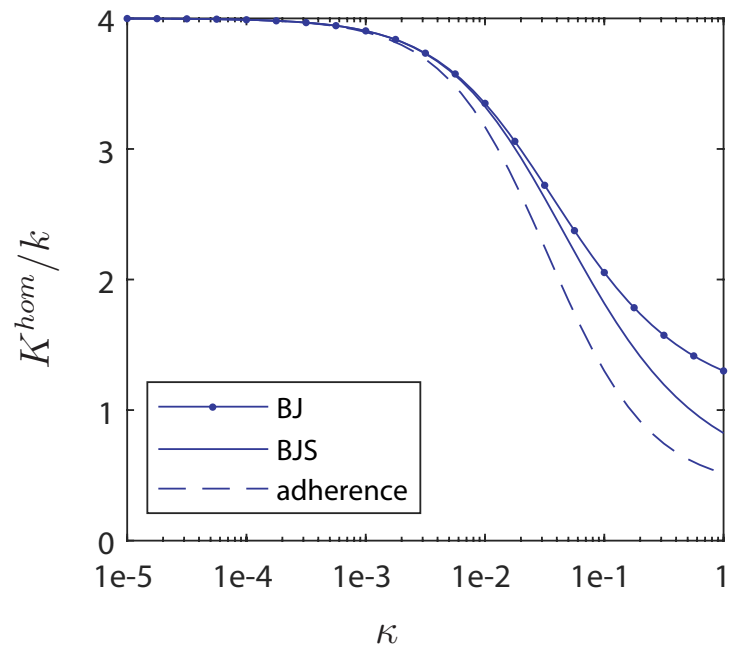

Fig. 7. Case of a spherical macropore. Variations of the dimensionless permeability $K^{\text {hom }} / k$ with $\kappa$ for $\delta=1$ and $f=0.5$. 


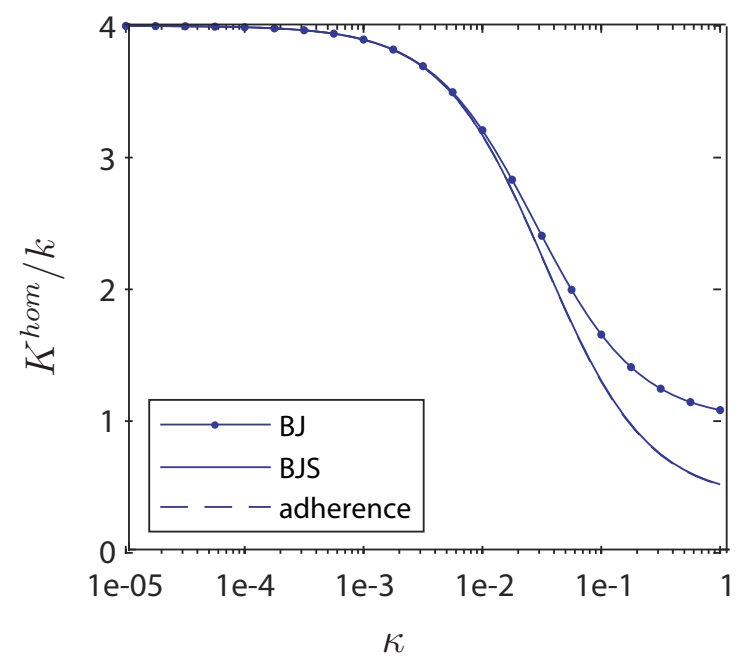

Fig. 8. Case of a spherical macropore. Variations of the dimensionless permeability $K^{\text {hom }} / k$ with $\kappa$ for $\delta=100$ and $f=0.5$.

Comparisons with finite element solutions are now provided to evaluate the accuracy of the analytic solutions. The calculations are performed on a squared unit cell containing a circular macropore. The periodic conditions are applied for the velocity and the pressure field on the boundary of the cell. The dimension of the unit cell is $L=10 \mu \mathrm{m}$. The calculations are performed by considering two values of the permeability of the microporous matrix: $k=10^{-15} \mathrm{~m}^{2}$ and $k=10^{-13} \mathrm{~m}^{2}$. We vary the radius of the macropore $a$, by doing so, we modify simultaneously the porosity $f=\pi a^{2} / L^{2}$ and coefficient $\kappa=k / a^{2}$.

Incompressible fluid flow problems generally contain velocity and pressure as the unknown variables and fall in the category of mixed formulations [49]. It was recognized that the solutions strongly depend upon the particular pair of velocity and pressure interpolations employed. The spaces of discretization must satisfy the inf-sup condition or the Ladyhenskaya-Babuska-Brezzi condition (see Brezzi [16]). Following [28], we use a mixed variational procedure with the Raviart-Thomas elements for Darcy region and MINI element for the Stokes region and the periodic boundary conditions are accounted with Lagrange multipliers. More details can be also found in [33].

The variation of the effective permeability with the radius of the macropore is represented on figs 9 and 10 for $k=10^{-15}$ and $k=10^{-13}$ respectively. The finite elements $(\mathrm{FE})$ solutions are compared with the analytic expressions in (61). The slip coefficient is $\delta=1$. A good agreement between the FE solutions and the analytic estimates of the effective permeability is observed. Differences are however observed for the higher values of the macropore radius that is probably attribuable to the shape of the unit cell: squared for the FE solution and circular for the analytic solutions. Also the differences could be attributed to the kind of boundary conditions, periodic for the squared unit cell and Dirichlet for the hollow cylinder. 


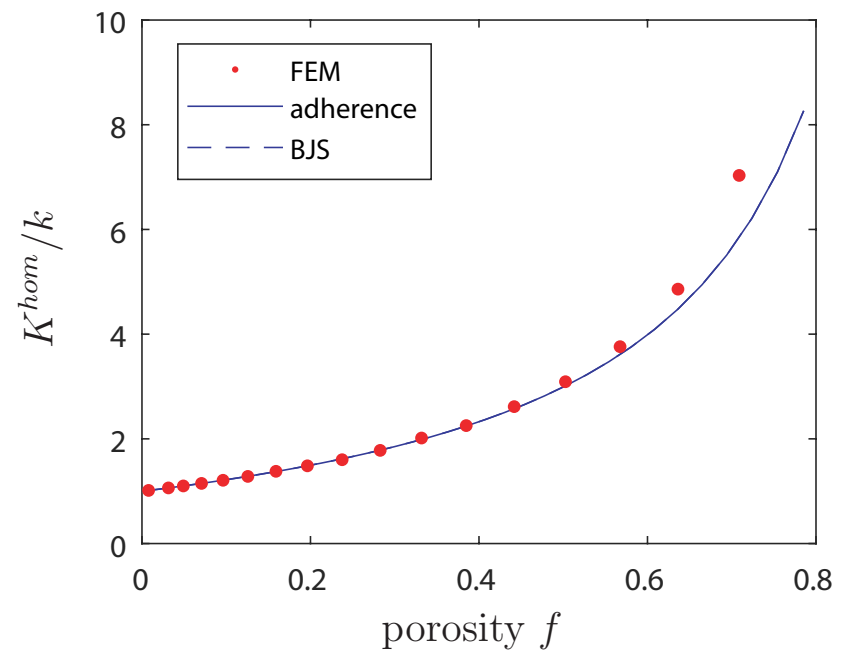

Fig. 9. Case of a cylindrical macropore. Variations of the effective permeability with the porosity for $k=10^{-15}$ and $\delta=1$. Comparison between FE solutions and the analytic expressions.

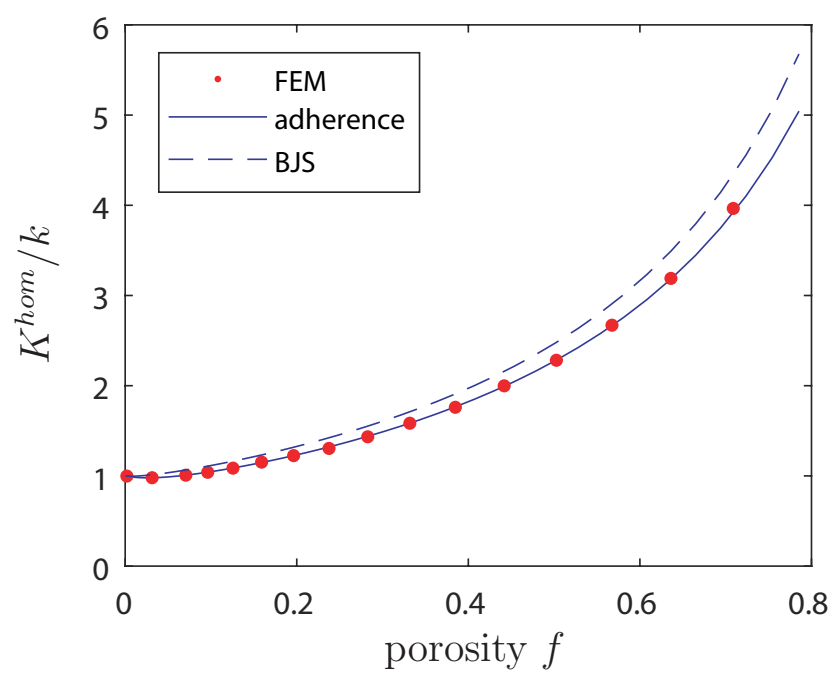

Fig. 10. Case of a cylindrical macropore. Variations of the effective permeability with the macropores radius for $\kappa=10^{-13}$ and $\delta=1$. Comparison between $\mathrm{FE}$ solutions and the analytic expressions.

\subsection{Approximation with an equivalent Darcy medium}

A simplification of the homogenization problem consists to replace the Stokes region by an equivalent Darcy medium with the permeability $k^{\prime}$. This simplification has been used in various works to determine the effective permeability of doubly porous solids with applications to fractured petroleum reservoir $[26,21,2]$. By combining two Darcy media, the boundary value problem use 
the same equations for both regions and the problem fall into classic homogenization boundary value problems. Indeed, the Darcy problem is equivalent to the thermal conduction and various results coming from the homogenization theory can then be used. From a numerical point of view, the problem is strongly simplified by considering two Darcy media. The numerical implementation of the coupled Darcy-Stokes problem involves mixed variational formulations and specific interpolation functions which satisfy to the inf-sup BBL condition. This has been already discussed in the previous section.

Now, the use of an equivalent Darcy medium also raise the question of the choice of $k^{\prime}$. It can be identified from the exact solutions derived in this paper. Consider a composite cylinder or sphere with two Darcy media. The core has the permeability $k^{\prime}$ and the surrounded matrix has the permeability $k$. We still consider the boundary conditions given by 13 . The solutions can be found in the book of Milton (established for the thermal conduction) and the resulting macroscopic permeability is:

$$
K^{h o m}=k\left\{1+\frac{2 f(c-1)}{(1-f) c+1+f}\right\}
$$

for the cylindrical shape and:

$$
K^{h o m}=k\left\{1+\frac{3 f(c-1)}{(1-f) c+2+f}\right\}
$$

for the spherical shape and where the coefficient $c$ is given by:

$$
c=\frac{k^{\prime}}{k}
$$

where $c$ represents the contrast between the two permeabilities (that of the macropore and that of the bulk microporous solid). Regarding first the case of a cylindrical macropore, the equivalent permeability is determined by comparing Eq. (64) to Eqs (59), (59) and (59). This leads to:

$$
\begin{array}{ll}
k^{\prime}=\frac{a^{2}}{2}\left(1+2 \kappa+\frac{2 \sqrt{\kappa}}{\delta}\right) & \text { BJ model } \\
k^{\prime}=\frac{a^{2}}{2}\left(1+\frac{2 \sqrt{\kappa}}{\delta}\right) & \text { BJS model } \\
k^{\prime}=\frac{a^{2}}{2} & \text { Adherence model }
\end{array}
$$

For the spherical macropore, the equivalent permeability is: 


$$
\begin{array}{ll}
k^{\prime}=\frac{a^{2}}{6}\left(1+6 \kappa+\frac{3 \sqrt{\kappa}}{\delta}\right) & \text { BJ model } \\
k^{\prime}=\frac{a^{2}}{6}\left(1+\frac{3 \sqrt{\kappa}}{\delta}\right) & \text { BJS model } \\
k^{\prime}=\frac{a^{2}}{6} & \text { Adherence model }
\end{array}
$$

The equivalent permeability is proportional to the square of the radius of the macropore. For the BJ and BJS models, the equivalent permeability also depends on the permeability of the microporous matrix and of the slip coefficient $\delta$. We observe that the equivalent permeability $k^{\prime}$ is different for a cylindrical and a spherical macropore. This suggests that the equivalent permeability depends on the shape of the macropore.

The present results differs from that of Markov et al. [34] who obtained (for the BJS interface model), the following expressions for the equivalent permeability:

$$
\begin{array}{ll}
k^{\prime}=\frac{a^{2}}{2}\left(1-\frac{3 \sqrt{\kappa}}{\delta}\right) & \text { cylinder } \\
k^{\prime}=\frac{a^{2}}{6}\left(1-\frac{4 \sqrt{\kappa}}{\delta}\right) & \text { sphere }
\end{array}
$$

These results has been derived by considering an isolated macropore embedded in an infinie microporous matrix. These expressions are different to that obtained in the present paper. Since the equivalent permeability is independent of the first porosity $f$, it would be expected to obtain the same results for $k^{\prime}$. Moreover the equivalent permeability obtained by Markov et al. [34] is negative if $\delta>3 \sqrt{\kappa}$ for the cylinder and if $\delta>4 \sqrt{\kappa}$ for the sphere. This can lead to some non physical results that are illustrated on Fig. 11. The effective permeability corresponding to spherical macropores is represented as function of the slip coefficient $\delta$ for $\kappa=10^{-2}$ and for two values of the porosity, namely $f=0.1$ (on the figure at the left) and $f=0.5$ (on the right). On these figures, we compare the estimate obtained in the present paper with the BJS model and the estimate derived by Markov et al. [34]. The results of the present paper show a very low influence of the slip coefficient on the effective permeability in the range $[0,1]$ while, the estimate of Markov et al. [34] has a singularity in that range. This singularity is observed when the denominator in Eq. (65) is null, that corresponding to $c=-(2+f) /(1-f)$ that is possible only if the equivalent permeability is negative. When the equivalent permeability is positive, $c>0$, no singularity could be noted for the effective permeability. 


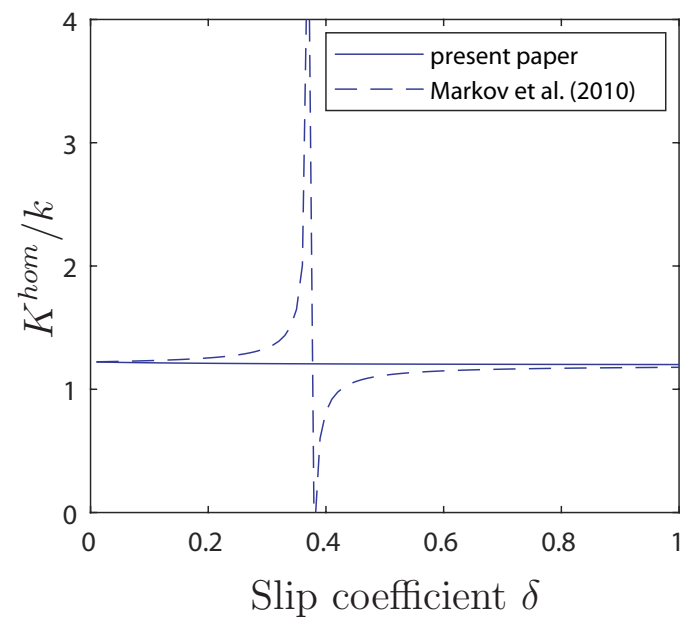

Fig. 11. Case of a spherical macropore. Variations of $K^{\text {hom }} / k$ as function of the slip coefficient $\delta$ for $\kappa=10^{-2}$ and $f=0.1$. Comparison between the Markov et al. (2010) estimate and that provided in the present paper with the BJS model.

\section{Conclusion}

The permeability of doubly porous solids with cylindrical and spherical macropores has been derived in a homogenization framework. The approach consists to solve a RVE problem with prescribed macroscopic pressure gradient at its boundary. In the RVE, we solve the coupled Darcy-Stokes problem and we use different interface conditions between the darcy and the stokes region (BeaversJoseph, Beavers-Joseph-Saffman and adherence conditions). Close form solutions are derived by considering a composite cylinder or sphere models. The results show that the interface model has no influence on the macroscopic permeability when there is a strong scale separation between the two porosities. At smaller scale separation, the choice of the interface model, and the value of the slip coefficient has a significant influence on the macroscopic permeability. The analytic estimates have been compared with FE solutions and show a good accuracy. Finally, an equivalent permeability for the Stokes region is identified. The results showed that this equivalent permeability depends on the shape of the macropore (cylindrical or spherical) and on the choice of the interface model. Such results are however not applicable to fractured porous solids, the fractures would depicted by considering ellipsoidal shapes.

\section{Acknowledgment}

This work has benefited from a French government grant by ANR within the frame of the national program Investments for the Future ANR-11-LABX022-01 (LabEx MMCD project). 
The authors declare that they have no conflict of interest.

\section{A Solution with prescribed velocity on the boundary}

\section{A.1 The cylindric macropore}

Consider first the case of the in plane solution. The boundary condition (14) reads in the cylindrical frame:

$$
v_{r}=V_{1} \cos (\theta)+V_{2} \sin (\theta)
$$

Only the case $V_{1} \neq 0$ and $V_{2}=0$ is considered.

The general expressions for the velocity and pressure fields with prescribed macroscopic velocity remains the same than that determined with the applied macroscopic pressure gradient $G_{1}$. These expressions are given in section (3) in which the expressions of the constants $A_{1}, B_{1}, A_{2}, C_{2}$ are different.

Only Eq. (35) in section (3), that corresponds to the applied pressure gradient, must be changed. Considering Eq. (A.1) with the expression of the velocity given by (30), we deduce that the boundary condition is $G(b)=V_{1}$ which also read:

$$
-\frac{k}{\mu}\left(A_{1}-\frac{B_{1}}{b^{2}}\right)=V_{1}
$$

Finally, the expressions of the constants are:

$$
\left.\begin{array}{l}
A_{1}=-\frac{\mu V_{1}}{k P}\left[2 a \mu+\alpha a^{2}+2 \alpha(1+\epsilon) k\right] \\
B_{1}=\frac{\mu V_{1}}{k P}\left(2 \mu a+\alpha a^{2}-2 \alpha k(1-\epsilon)\right) a^{2} \\
A_{2}=-\frac{8 k \alpha \mu V_{1}}{k P} \\
C_{2}=\frac{V_{1}}{P}\left(4 \alpha \epsilon k+4 a \mu+3 \alpha a^{2}\right)
\end{array}\right\}
$$

in which $Q$ is given by:

$$
P=\left[2 a \mu+\alpha a^{2}+2 \alpha \epsilon k\right](1+f)+2 \alpha k(1-f)
$$




\section{A.2 The spherical macropore}

In the case of a spherical macropore, the condition (53) must be replaced by:

$$
-\frac{k}{\mu}\left(A_{1}-2 \frac{B_{1}}{b^{3}}\right)=V_{1}
$$

The identification of the constants leads to:

$$
\begin{array}{r}
A_{1}=-\frac{\mu V_{1}\left(a^{2} \alpha+6 \alpha k(2+\epsilon)+3 a \mu\right)}{k P}, \\
B_{1}=\frac{\mu V_{1} a^{3}}{k P}\left(a^{2} \alpha+3 a \mu-6 \alpha(1-\epsilon) k\right), \\
C_{1}=\frac{3 V_{1}}{P}\left(3 a \mu+2 a^{2} \alpha+6 \alpha \epsilon k\right), \\
A_{2}=-\frac{30 \mu V_{1} \alpha}{P}
\end{array}
$$

with:

$$
P=\left[\alpha a^{2}+3 a \mu+6 k \alpha \epsilon\right](1+2 f)+12 \alpha k(1-f)
$$

\section{References}

[1] Allaire G. Homogenization of the Stokes flow in a connected porous medium. Asymptotic Analysis. 1989; 2 (3):203-222,

[2] Arbogast T., Douglas J., Hornung U. Derivation of the double porosity model of single phase flow via homogenization theory. Siam J. Math. Anal. 1990; 21(4):823-836.

[3] Arbogast T., Lehr H.L. Homogebnization of a Darcy-Stokes systen modeling vuggy porous media. Comput. Geosciences. 2006, 10(3):291-302.

[4] Arbogast T., Brunson D.S. A computatonal method for approximating a DarcyStokes system governing a vuggy porous medium. Comput. Geosciences. 2007, 11(3):207-218.

[5] Auriault J.L., Sanchez-Palencia E. Study of macroscopic behavior of a deformable porous medium. J. Méca. 1977; 16 (4):575-603.

[6] Auriault, J.L., Boutin, C. Deformable porous media with double porosity. Quasi-statics. I: Coupling effects. Transp Porous Med (1992) 7: 63.

[7] Auriault, J.L., Boutin, C. Deformable porous media with double porosity. Quasi-statics. II: Memory effects 
[8] Auriault, J.L., Boutin, C. Deformable porous media with double porosity III: Acoustics. Transp Porous Med (1994) 14: 143.

[9] Auriault J.L. About the Beavers and Joseph boundary condition. Transp. Porous Med. 2010, 83:257-266.

[10] Beavers G.S., Joseph D.D. Boundary condition at a naturally permeable wall J. Fluid Mech. 1967; A (1):27-34.

[11] Boutin C., Royer P. and Auriault J.L. Acoustic absorption of porous surfacing with dual porosity. Int. J. Solids Structures. 34:4709-4737, 1998.

[12] Boutin C. Study of permeability by periodic and self consistent homogenization. Eur. J. Mech. A/solids 19:603-632, 2000.

[13] Brezzi F. and Fortin M. Mixed and Hybrid Finite Element Methods, Springer, New York, 1991.

[14] Brinkman H.C., A calculation of the viscous force exerted by a flowing fluid on a dense swarm of particles. App. Sci. Res. 1949; A (1):27-34.

[15] Brinkman H.C., On the permeability of media consisting of closely packed porous particles. App. Sci. Res. 1949; A (1):81-86.

[16] Brezzi F., On the existence, uniqueness and approximation of saddle-point problems arising from Lagrangian multipliers, RAIRO, 8 :129-151, 1974.

[17] Budiansky B., O'Connell R.J. Elastic moduli of a cracked solid. Int. J. Solids Struct. 12(2):81-97, 1976.

[18] Celle P., Drapier S., Bergheau J.-M. Numerical modelling of liquid infusion into fibrous media undergoing compaction. Eur. J. Mech. A/Solids. 27:647-661, 2008 .

[19] Chen N., Gunzburger M., Wang Xiaoming W. Asymptotic analysis of the differences between the Stokes-Darcu system with different interface conditions and the Stokes-Brinkman system. J. Math. Anal. Appl. 368:658-676, 2010.

[20] Correa M.R., Loula A.F.D. A unified mixed formulation naturally coupling Stokes and Darcy flows. Comput. Methods Appl. Mech. Engrg. 198:2710-2722, 2009 .

[21] de Swaan A. Analytic solutions for determining naturally fractured reservoir properties by well testing. Soc. Petroleum Engrs. J. 16:117-122, 1976.

[22] Discacciati M., Quarteroni A., Valli A. Robin-Robin domain decomposition methods for the Stokes-Darcy coupling. SIAM Journal on Numerical Analysis. 45(3):1246-1268, 2007.

[23] Happel J., Viscous flow relative to arrays of cylinders. AIChEF J. 5(2):174-177, 1959.

[24] Jager W., Mikelic A., On the interface boundary condition of Bevears, Joseph and Saffman. SIAM J. Appl. Math. 60(4):1111-1127, 2009. 
[25] Karper T., Mardal K.-A., Winther R. Unified finite element discretizations of coupled darcyÜstokes flow. Num. Meth. Part. Diff. Eq. 25(2):311-326, 2009.

[26] Kazemi H., Pressure transient analysis of naturally fractured reservoirs with uniform fracture distribution. Soc. Petroleum Engrs. J. 9:451-462, 1969.

[27] Kuwabara S., The forces experienced by randomly distributed parallel circular cylinders or spheres in a viscous flow at small Reynolds Numbers. J. Phys. Soc. Jpn.. 14(4):527-532, 1959.

[28] Layton W.J., Schieweck F., Yotov I., Coupling fluid flow with porous media flow. SIAM J. Appl. Math. 60:2195-2218, 2000.

[29] Lequang H., He Q.C. Variational principles and bounds for elastic inhomogeneous materials with coherent imperfect interfaces. Mech. Mater. 40:865-884, 2008.

[30] Levy T. Fluid flow through an array of fixed particles. Int. J. Engng. Sci. 21(1):11-23, 1983.

[31] Ly H.B., Le Droumaguet B., Monchiet V., Grande D. Designing and modeling doubly porous polymeric materials. European Physical Journal. Eur. Phys. J. 224(9):1689-1706, 2015.

[32] Ly H.B., Le Droumaguet B., Monchiet V., Grande D. Facile fabrication of doubly porous polymeric materials with controlled nano- and macro-porosity. Polymer. 78(5):13-21, 2015.

[33] Ly H.B., Functionalized doubly porous polymeric materials : design and modeling. PhD, 2015.

[34] Markov M., Kazatchenko E., Mousatov A., Pervago E. Permeability of the Fluid-Filled Inclusions in Porous Media. Transp. Porous Med. 84:307-317, 2010.

[35] Nied D.A., The Beavers-Joseph Booundary condition and related Matters: A historical and critical note. Transp. Porous Med.. 78:537-540, 2009.

[36] Olny X. and Boutin C. Acoustic wave propagation in double porosity media. J. Acoust. Soc. Am. 114(1):73-89, 2003.

[37] Ochoa-Tapia J.A., Whitaker S. Momentum transfer at the boundary between a porous medium and a homogeneous fluid. I. theorethical development. Int. J. Heat Mass Transf.. 38(14):2635-2646, 1995.

[38] Ochoa-Tapia J.A., Whitaker S. Momentum transfer at the boundary between a porous medium and a homogeneous fluid. II. comparison with experiment. Int. J. Heat Mass Transf.. 38(14):2647-2655, 1995.

[39] Rasoulzadeh M., Kuchuk F.J. Effective Permeability of a Porous Medium with Spherical and Spheroidal Vug and Fracture Inclusions Transp Porous Med. 116:613Ü644, 2017.

[40] Royer P., Auriault J.L. and Boutin C. Macroscopic modeling of double-porosity reservoirs. J. Petroleum Sc. Engrg. 16:187-202, 1996. 
[41] Saffman P.G., On the boundary condition at the interface of a porous medium. Stud. Appl. Math. 1: 93-101, 1971.

[42] Sahraoui M., Kaviany M., Slip and no slip velocity boundary conditions at interface of porous plain media. Int. J. Heat Mass Transfer. 35(4):927-943, 1992.

[43] Sanchez-Palencia E. Nonhomogeneous Media and Vibration Theory, Lecture Notes in Physics, Springer, Berlin. 127, 1980.

[44] Silva G. and Ginzburg I. StokesÜBrinkmanÜDarcy solutions of bimodal porous flow across periodic array of permeable cylindrical inclusions: cell model, lubrication theory and LBM/FEM numerical simulations. Transp. Porous Media. 2016, vol. 111(3), pp. 795-825.

[45] Stechkina I.B., Drag of porous cylinders in a viscous fluid at low Reynolds numbers. Fluid. Dyn. 14(6):912, 1979.

[46] Urquiza J.M., Dri D.N., Garon A., Delfour M.C. Coupling Stokes and Darcy equations. Appl. Num. Math. 58:525-538, 2008.

[47] Valdes-Parada F.J., Goyeau B., Ochoa-Tapia J.A. Jump momentum boundary condition at a fluid-porous dividing surface: derivation of the closure problem. Chem. Eng. Sci. 62:4025-4039, 2007.

[48] Whitaker S. Flow in porous media I: A theoretical derivation of Darcy's law. Transport in Porous Media. 1(1):3-25, 1986.

[49] Zienkiewicz O.C., Taylor R.L. The Finite Element Method, 4th edition, Vol. I (McGraw-Hill, 1981). 